\title{
On the Propagation and Structure of Ionization Fronts
}

\author{
F. A. Goldsworthy \\ Manchester University, Manchester, England
}

\section{INTRODUCTION}

$\mathrm{T}$ HE problem discussed here is that of determining the motion of a cloud of neutral atomic hydrogen gas, when it is subjected to ionizing radiation from a star embedded in it. Initially the gas is in gravitational equilibrium at a constant temperature of about $100^{\circ} \mathrm{K}$. It is supposed that at time $t=0$ the star suddenly begins to radiate with a certain intensity, which remains constant thereafter. Part of the surrounding gas will be ionized and an ionization front (separating the ionized gas from the neutral gas) will move outwards into the neutral gas. A shock wave may also propagate ahead of the ionization front into the neutral gas. There will therefore be two regions to consider-a region of ionized gas (HII region) and a region of neutral gas (HI region) in which there may be a shock.

\section{EQUATIONS OF MOTION IN THE HII REGION}

(i) Outward flux of quanta,

$$
\frac{\partial}{\partial r}\left(J r^{n}\right)=-\frac{\alpha}{M} J \rho(1-x) r^{n},
$$

where $J$ is the number of photons crossing unit area per second, $x$ is the fraction of ionized atoms, $r$ is the distance from the star, $\alpha=\left(6 \times 10^{-18} \mathrm{~cm}^{2}\right)$ is the absorption coefficient for ionizing radiation, $M$ is the mass of a hydrogen atom, $\rho$ is the density of the gas, and $n$ is 1 if cylindrical symmetry exists and 2 for spherical symmetry. Re-emission of quanta in the gas itself has been neglected in Eq. (1) in order to preserve radial symmetry.

At the star, $r=R_{*}, J=J_{*}$ for all time.

(ii) Ionization balance equation,

$$
\frac{D x}{D t}=\alpha J(1-x)-\frac{\beta \rho x^{2}}{M},
$$

where $D / D t=\partial / \partial t+u \partial / \partial r, u$ is the radial velocity of the gas, $\beta\left(=3 \times 10^{-10} T^{-1} \mathrm{~cm}^{3} \mathrm{sec}^{-1}\right)$ is the recombination coefficient, and $T$ is the temperature. The first term on the right-hand side represents the production of ions by the absorption of radiation from the star, and the second term represents the loss of ions which recombine with electrons in the HII region.

(iii) Equation of state,

$$
p=(k / M) \rho(1+x) T,
$$

where $k$ is Boltzmann's constant. (iv) Equation of momentum,

$$
\frac{D u}{D t}=-\frac{1}{\rho} \frac{\partial p}{\partial r}-\frac{G \mathscr{T}}{r^{n}}
$$

where $p$ is the pressure, $\mathfrak{T C}$ is the mass of the star or twice the mass per unit length of the axis along which we suppose a group of stars to be distributed (i.e., approximate cylindrical symmetry exists).

(v) Equation of continuity,

$$
\frac{\partial \rho}{\partial t}+\frac{1}{r^{n}} \frac{\partial}{\partial r}\left(r^{n} \rho u\right)=0
$$

(vi) Equation of energy,

$$
\begin{aligned}
\frac{D}{D t}\left(\frac{3}{2} \frac{p}{\rho}\right)+p \frac{D}{D t}\left(\frac{1}{\rho}\right) & =\frac{\alpha}{2} J(1-x) Q_{0}{ }^{2} \\
- & \frac{3 \rho x^{2} k T}{2}-10^{-27} \frac{\rho x^{2}}{M^{2}}(T-7000),
\end{aligned}
$$

where $(1 / 2) M Q_{0}{ }^{2}$ is the amount of energy left over on the average in each ionization. The second and third terms on the right-hand side of Eq. (6) represent the loss of heat of the gas by recombination of ions with electrons and by the excitation of $\mathrm{O}^{+}$ions, respectively. The latter term is absent if $T<7000^{\circ} \mathrm{K}$.

\section{EQUATIONS OF MOTION IN THE HI REGION}

These are the equations of momentum and continuity as given in the foregoing, and the energy equation (6), with the right-hand side zero, if the cooling of the neutral gas behind the shock wave is assumed to be unimportant.

Initially the density, $\rho_{0}(r)$, of the neutral gas is given by

$$
\frac{k T_{0}}{M} \frac{1}{\rho_{0}} \frac{\partial \rho_{0}}{\partial r}=-\frac{G \Re}{r^{n}}
$$

where $T_{0}$ is the temperature of the gas.

\section{CONDITIONS AT THE IONIZATION FRONT $\left(r=R_{i}(t)\right)$}

The region in which the neutral gas becomes ionized, and therefore where absorption is the greatest, is represented mathematically as a discontinuity, which we call an ionization front. Suffixes $i$ and $n$ will be 
used to denote data on the ionized and neutral sides of the front. Then, provided that there is not sufficient time for recombination to take place across the front, it can be shown that

$$
\begin{aligned}
J_{i} & =\left(U_{i}-u_{i}\right) \rho_{i} x_{i} M^{-1}, \\
\rho_{n}\left(U_{i}-u_{n}\right) & =\rho_{i}\left(U_{i}-u_{i}\right), \\
p_{n}+\rho_{n}\left(U_{i}-u_{n}\right)^{2} & =p_{i}+\rho_{i}\left(U_{i}-u_{i}\right)^{2}, \\
\frac{5}{2} \frac{p_{n}}{\rho_{n}}+\frac{1}{2}\left(U_{i}-u_{n}\right)^{2}+\frac{1}{2} x_{i} Q_{0}{ }^{2} & =\frac{5}{2} \frac{p_{i}}{\rho_{i}}+\frac{1}{2}\left(U_{i}-u_{i}\right)^{2},
\end{aligned}
$$

where $U_{i}$ is the velocity of the front.

A solution of some rather complicated partial differential equations subject to certain boundary conditions is required. Several difficulties are immediately apparent, among them is that of knowing the initial flow pattern. For instance, one does not know whether a shock will occur in the neutral gas or perhaps even in the ionized region, and the difficulty of solving partial differential equations without this information is well known. We therefore look for a similarity solution, i.e., a solution depending on one independent variable only, so that the system of partial differential equations will reduce to a system of ordinary differential equations, which can be solved easily. The similarity solution will be applicable when the size of the star is not a relevant factor in determining the motion of the gas. The star is thus to be regarded as a point or line source or radiation. The strength of the source we shall define as the total photon output and this must be equal to the total photon output of the star, i.e.,

$$
\operatorname{Lim}_{r \rightarrow 0} J r^{n}=J * R *^{n}
$$

Several restrictions are immediately placed upon the form of the similarity solution.

(i) The temperature $T *$ of the star is constant, and we shall assume that the spectral quality of the radiation field remains constant also, so that $Q_{0}$, which is a typical velocity in the problem, is constant. Hence the similarity variable $\eta$ must be $t / r$, i.e., the ionization front moves out with constant speed.

(ii) Equation (8) means that $\rho / J$ must remain a constant at the ionization front, and hence $\rho / J$ must be a function of $\eta$ only.

(iii) $J r^{n}$ must be a function of $\eta$ only in order that $J \boldsymbol{r}^{n}$ will tend to a finite limit as $\eta \rightarrow \infty$ (i.e., $\boldsymbol{r} \rightarrow 0$ ).

We now distinguish between the spherical and cylindrical cases.

\section{SPHERICAL IONIZATION FRONTS (I)}

Restrictions (ii) and (iii) imply that $\rho r^{2}$ must be a function of $\eta$ only. This is unfortunate as it means that in order that a similarity solution may be found, gravitation must be neglected and the effect of recombination must be assumed to be negligible, i.e., the HII region must be regarded as being completely ionized. The cooling caused by the excitation of $\mathrm{O}^{+}$ions has also to be ignored. Equations (4) to (6) then reduce to the well-known equations for fluid flow. To these we add $J r^{n}=J_{*} R^{n}$ and $x=1$. These equations can be solved and the flow pattern determined for given values of the parameters involved in the problem. If the initial density distribution in the neutral gas is given by $\rho_{0}=\omega_{0} r^{-2}$ then $\omega_{0}$ is one parameter. The other significant parameter is $T *$.

It can be shown that, if

$$
\omega_{0}=M J * R *^{2} Q_{0}{ }^{-1} 2^{-\frac{1}{1}},
$$

which is a function of $T_{*}$, then an ionization front (similar to a strong detonation) will propagate by itself into the neutral gas with a speed $U_{i}=Q_{0} 2^{\frac{1}{t}}$. If $\omega_{0}>\left(M J * R *^{2}\right) /\left(Q_{0} 2^{\frac{1}{2}}\right)$ then a shock wave propagates ahead of the ionization front, thus disturbing the neutral gas before ionization takes place. Results indicate that for a given type of star the ionization front travels slower in this case than when the equality sign held. For the case when $\omega_{0}<\left(M J_{*} R *^{2}\right) /\left(Q_{0} 2^{\frac{1}{t}}\right)$, the flow pattern is not too clear. A possible configuration is one in which we have a shock traveling ahead of the ionization front and a second shock in the ionized region. Results indicate that for a given type of star the speed of propagation is higher in this case than in the previous two cases. However, further investigations are necessary before the results for this case can become conclusive.

From the similarity solution one can plot the temperature distribution in the ionized region. It is found that the temperature goes to zero at the origin (i.e., at the source). This is the outcome of neglecting recombination and subsequent re-ionization, which will maintain the temperature at a finite value. Thus to get a more accurate picture we need to consider in some way the effect of recombination of ions with electrons. For mathematical reasons this can best be done for a problem in which cylindrical symmetry exists.

\section{CYLINDRICAL IONIZATION FRONTS}

Before considering the propagation of cylindrical fronts, brief mention will be made of the cylindrical model which has been considered. It is supposed that stars are distributed along a line at equal intervals of distance, and that the total photon output of the "cylindrical" star is equal to the sum of the outputs of the individual stars.

In the cylindrical case restrictions (ii) and (iii) imply that $\rho r$ must be a function of $\eta$ only. This means that the initial distribution of the density must be given by $\rho_{0}=\omega_{0} r^{-1}$. This density distribution is compatible with Eq. (8) only if $G \mathscr{T M} M / k T_{0}=1$, where, as mentioned before, $\mathfrak{N}$ now is twice the mass of the "cylindrical" star per unit length. Inserting numerical values we obtain : $\frac{1}{2} \mathfrak{T}=6.2 \times 10^{14} T_{0} \mathrm{~g} / \mathrm{cm}$. Since this is a rather 
TABLE I.

\begin{tabular}{crrrrr}
\hline \hline Star & \multicolumn{1}{c}{ B2 } & \multicolumn{1}{c}{ B0 } & \multicolumn{1}{c}{ O9 } & \multicolumn{1}{c}{ O7 } & \multicolumn{1}{c}{ O5 } \\
\hline$T *\left({ }^{\circ} \mathrm{K}\right)$ & 20000 & 25000 & 32000 & 50000 & 80000 \\
$T_{\mathrm{c}}\left({ }^{\circ} \mathrm{K}\right)$ & 7461 & 7689 & 7994 & 8719 & 9788 \\
\hline \hline
\end{tabular}

special value, it is better to neglect gravitation, as was done in the spherical case.

Substituting the appropriate similarity forms for the velocity, pressure, etc., in Eqs. (1) to (6), six ordinary differential equations can be obtained, and gravitation, recombination, etc., can all be taken into account. These equations can then be solved. However, there arises the difficulty of fixing the values of the two parameters $\omega_{0}$ and $T *$ corresponding to a particular flow pattern-an ionization front traveling by itself, say. It turns out that a series of test runs must be made on a computing machine before one can obtain the required information. One can however derive an extremely accurate result theoretically without much labor. For the basic approximation there is no need to introduce similarity into the problem at all, and the approximation holds also in the spherical and plane cases.

Eliminating $J$ between Eqs. (2) and (6), we obtain

$$
\begin{aligned}
& \frac{D}{D t}\left(\frac{3}{2} \frac{p}{\rho}\right)+p \frac{D}{D t}\left(\rho^{-1}\right)-\frac{Q_{0}{ }^{2}}{2} \frac{D x}{D t} \\
& \quad=\frac{\beta \rho x^{2}}{M}\left(\frac{1}{2} Q_{0}{ }^{2}-\frac{3}{2} \frac{k T}{M}\right)-10^{-27} \frac{\rho x^{2}}{M^{2}}(T-7000) .
\end{aligned}
$$

Using Eq. (3), this can be rewritten in the form

$$
\begin{array}{r}
\frac{3}{2} T^{-\frac{D T}{D t}}-\rho^{-1} \frac{D \rho}{D t}-\left(\frac{M Q_{0}{ }^{2}}{2 k T}-\frac{3}{2}\right)(1+x)^{-1} \frac{D x}{D t} \\
=0.4 \times 10^{13} \rho x^{2}(1+x)^{-1}\left[10^{27} \beta k\left(\frac{M Q_{0}^{2}}{2 k T}-\frac{3}{2}\right)\right. \\
-(1-7000 / T)] .
\end{array}
$$

If we take a time-scale of a million years, so that $t$ is expressed in this quantity as unit, the right-hand side must be multiplied by $3 \times 10^{13}$ and the numerical coefficient becomes $1 \times 10^{26}$. Hence, even with densities

\begin{tabular}{|c|c|c|c|c|c|}
\hline Star & B2 & BO & O9 & $\mathrm{O} 7$ & 05 \\
\hline$\omega_{0}(\mathrm{~km} / \mathrm{sec})$ & $\begin{array}{r}0.65 \times 1 \\
23\end{array}$ & $\begin{array}{l}70 \times 10 \\
23.5\end{array}$ & $\begin{array}{l}4 \times 1 \\
24\end{array}$ & $\begin{array}{c}53 \times 1 \\
25\end{array}$ & $\begin{array}{l}68 \times 10^{-} \\
26.5\end{array}$ \\
\hline
\end{tabular}
of a few hydrogen atoms per $\mathrm{cm}^{3}$ the quantity inside the square brackets on the right-hand side of the equation is multiplied by a very large factor.

TABLE II.
Thus, unless $T$ is near the value $T_{c}$, for which the term in the square brackets is zero, at least one term on the left-hand side of Eq. (15) must be large; we rule out a large rate of change of $x$ and $\rho$ and consider the rate of change of the temperature. It can be shown that if $T \lesseqgtr T_{c}$, then $D T / D t \geqq 0$. Thus, if at any point of the ionized region the temperature is very much different from $T_{c}$, the temperature will increase or decrease to it in a short interval of time. The effect, therefore, of recombination and subsequent re-ionization together with the excitation of $\mathrm{O}^{+}$ions is to maintain the ionized region at a constant temperature very near to $T_{c}$. Typical values of $T_{c}$ for the various types of stars are shown in Table I. Values of $T_{*}$ given in Table I are those proposedb y Kahn. ${ }^{1}$ Thus, instead of the rather complicated Eq. (6), we write $T=T_{c}$, and replace the temperature in Eqs. (1) to (5) by $T_{c}$. The analysis can be simplified further, for in the HII region, the gas will be nearly fully ionized, so that $x$ can be assumed approximately equal to 1 and $D x / D t$ can be neglected in Eq. (2). One notes, however, that the variation of $(1-x)$ must still be retained in the absorption term.

By making the above assumptions though, we must modify our ideas of the ionization front. We must now

TABLE III.

\begin{tabular}{lccccc}
\hline \hline$\omega_{0}$ & $0.88 \times 10^{-3}$ & $2.30 \times 10^{-3}$ & $0.73 \times 10^{-2}$ & $2.07 \times 10^{-2}$ & $0.92 \times 10^{-1}$ \\
\hline$U_{i}(\mathrm{~km} / \mathrm{sec})$ & 14.4 & 14.6 & 15.0 & 15.6 & 16.6 \\
$U_{s}(\mathrm{~km} / \mathrm{sec})$ & 16.5 & 16.7 & 17.1 & 17.8 & 19.0 \\
$T_{s}\left({ }^{\circ} \mathrm{K}\right)$ & 10370 & 10688 & 11112 & 12119 & 13605 \\
\hline
\end{tabular}

allow for the rapid cooling of the gas by recombination and the excitation of ions in the region of the ionization front, so that a particle of fluid having passed through the front almost at once is at a temperature $T_{c}$. This means that only the equations of continuity and momentum can be used to connect data on the two sides of the front. These turn out to be sufficient to solve the problem uniquely.

The solution that will be described is one based on similarity, for which the initial density distribution is given by $\rho_{0}=\omega_{0} r^{-1}$. Gravitation will be neglected since the thermal velocity is high.

It can be shown that, if $\omega_{0}$ and $T *$ are related by the equation

$$
\omega_{0}^{2}=M^{2} J * R * / 1.044 \beta,
$$

then an ionization front will propagate into the undisturbed neutral gas with a speed $\left(2 k T_{c} / 0.23 M\right)^{\frac{1}{2}}$. One notices that, taking into account the cooling of the gas and re-ionization, the velocity of propagation is proportioned to $\left(T_{c}\right)^{\frac{1}{2}}$, instead of $\left(T_{*}\right)^{\frac{1}{2}}$ as was found in Sec. 6. Table II gives the values of $\omega_{0}$ for each type of star for which the above description of the flow pattern

${ }^{1}$ F. D. Kahn, Bull. Astron. Soc. Neth. No. 456, 197 (1956). 


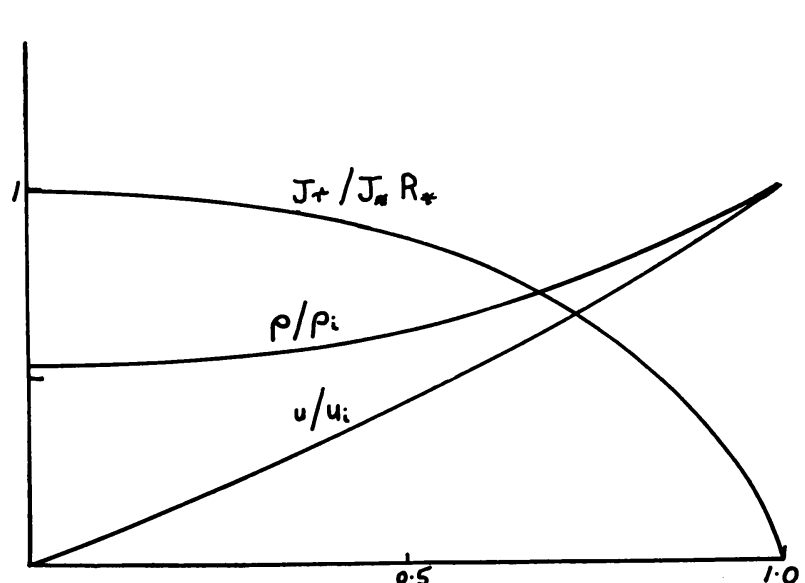

(a)

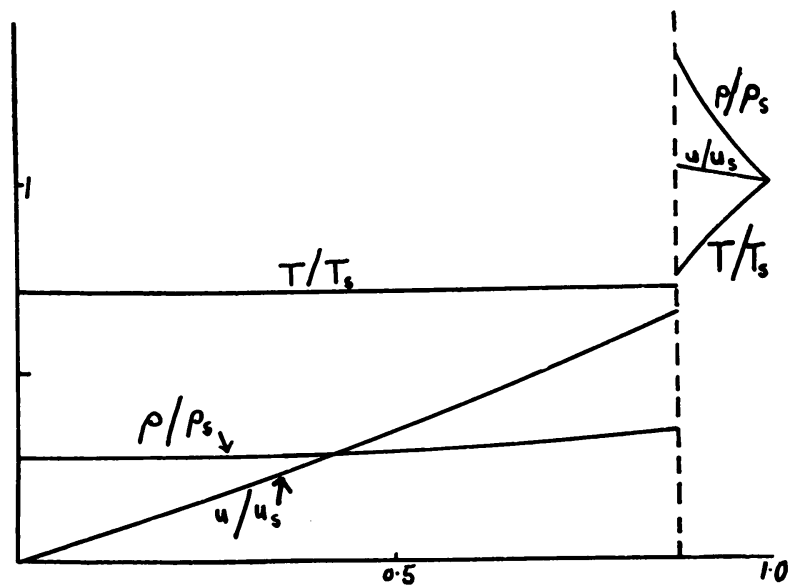

(b)

FIG. 1. (a) Velocity and density distributions for flow behind an ionization front traveling by itself. (b) Velocity, density, and temperature distribution for the case of an ionization front preceded by a shock.

would be applicable. Values of the photon output, etc. which have been used, are those quoted by Kahn. ${ }^{1}$

Table III gives typical value of $\omega_{0}$ for which a shock will move ahead of the ionization front. The shock speeds are given in the third row of Table II; $T_{s}$ is the temperature behind the shock. When $\omega_{0}$ is less than the value given by Eq. (16), then it seems likely that an "isothermal" shock will be present in the ionized region. The author is pursuing further investigations into this case.

In Fig. 1, the temperature, density, and pressure and velocity distributions are plotted for the above two cases. The absorption of the total number of photons $(J r)$ crossing unit length of a cylinder of radius $r$ is also illustrated in the first case. It shows that the absorption from the star is appreciable in the HII region, i.e., $J_{i} R_{i}<J_{*} R_{*}$. This fact, together with Eqs. (1) and (2), enables the following approximate integral relationship to be obtained

$$
J_{*} R_{*}=\frac{\beta}{M^{2}} \int_{0}^{R_{i}} \rho^{2} r d r
$$

from which $\omega_{0}$ can be determined quickly.

We have thus found that the rate of expansion of an ionized region into a medium whose initial density varies like $1 / r$ is of the order of $24 \mathrm{~km} / \mathrm{sec}$ and that the temperature of the ionized region is uniform and is about $8000^{\circ} \mathrm{K}$. The obvious question that now comes to mind is whether or not this latter approximation can be checked. This can easily be done in the ionized region itself by regarding Eq. (15) as giving the second approximation for the temperature. It turns out that the maximum change is very small indeed. The next check to be made is an answer to the question: How quickly does a fluid particle having entered the ionization front come to the temperature $T_{c}$ ? This will involve a study of the structure of an "ionization front."

\section{STRUCTURE OF AN IONIZATION FRONT}

For the sake of simplicity we consider the structure of an $R$-type ionization front which moves with supersonic speed relative to the undisturbed fluid ahead and with subsonic speed relative to the fluid behind (this is the type which occurs in the first case described in Sec. 6). It will be assumed that the action of viscosity and thermal conductivity is confined to a region of thickness small compared with the thickness of the ionization front, i.e., a shock wave may occur in the region of the ionization front. Now the above type of ionization front, which is similar to a strong detonation, can be regarded as an ionization front $[D$ type (Editor's note: cf. Pottasch, these proceedings) which moves with subsonic speed relative to the fluid ahead and behind] initiated by shock, i.e., in the same manner that a strong detonation is described in terms of a weak deflagration initiated by a shock. Through the shock, the pressure, density, temperature, and entropy are raised instantaneously; through the

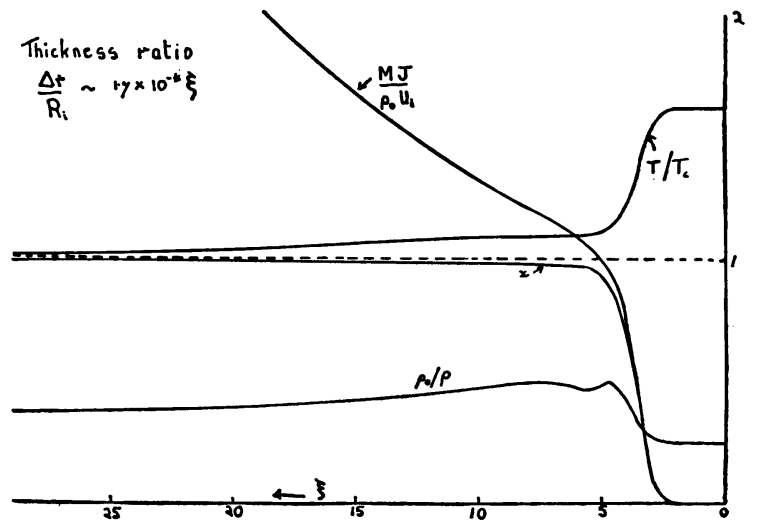

FIG. 2. Density ratio, $\rho_{0} / \rho$, fraction of ionized atoms, $x$, temperature ratio, $T / T_{\epsilon}$, and $W J / \rho_{0} u i$ plotted as a function of distance. 
subsequent ionization process the temperature decreases to the value $T_{c}$ and $x$ approaches 1 . In Fig. 2 this is illustrated for the case of an ionization front which is produced by the radiation from a B2-type star. The thickness of the ionization front is about $1 / 1000$ of the ionization front radius. The diagram also shows the effect of recombination to the rear, for here $x$ is very much different from $M J / \rho_{0} U_{i}$. Thus, the assumptions, which were made in Sec. 6 can be regarded as a valid approximation.

\section{SPHERICAL IONIZATION FRONTS (II)}

Finally, brief mention must be made of the form of the attack on the spherical problem, having found an approximate basis on which to work taking into account cooling. The previous result has shown that no longer is it true to say that at the ionization front the number of ions coming out of the front is equal to the number of photons entering, i.e., Eq. (8) is now not applicable.
This removes restriction (ii) in Sec. (4) and is replaced by the fact that absorption in the ionized region is appreciable so that

$$
J_{*} R^{2}=\frac{\beta}{M^{2}} \int_{0}^{R_{i}} \rho^{2} r^{2} d r
$$

corresponding to Eq. (17) for the cylindrical case. Since $J_{*} R *^{2}$ is constant, Eq. (18) shows that $\rho^{2} r^{3}$ must be a function of the similarity variable $t / r$. Thus, we can now solve the spherical problem, taking into account cooling and re-ionization in the HII region, for the case when the initial density distribution is $\rho_{0}=\omega_{0} / r^{\frac{3}{2}}$. The results obtained are essentially the same as given in Tables II and III, except that the order of magnitude of $\omega_{0}$ is changed, for instance $\omega_{0}=0.65 \times 10^{-3}$ for a B2-type cylindrical star becomes $\omega_{0}=8.3 \times 10^{\frac{1}{1}}$ in the spherical case. The speeds of propagation are of the same order.

\section{DISCUSSION}

Editor's Note: Since the papers by Pottasch, Kahn, and Goldsworthy treat the same subject, the discussion on them has been combined.

E. Schatzman, Institut d'Astrophysique, Paris, France: If I understand, you have supposed that in the $\mathrm{HI}$ region the wave is propagating to infinity and that there is no reflection. This acts as a damping effect because the energy which is put in by your corrugated surface is going away. But, if you had some other boundary condition, would you not have some energy fed back in your oscillatory process that would decrease the time of amplification?

F. D. KAHN, University of Manchester, Manchester, England: This may be the case for some values of the parameters, but the ones which are most interesting with temperatures of the stars of the order of $50000^{\circ}$, gas temperatures on the order of $100^{\circ}$, and the star doubling its intensity in a million years, lead to the result that the whole motion set up by the corrugations takes place in a region which is only about one hundredth of a parsec thick, and hardly anything goes on further ahead of the ionization front. Thus, in considering slabs of matter which are more than a hundredth of a parsec thick, one is probably justified in putting the far boundary at infinity.

\section{E. SchATZMAN : There is no progressive wave?}

F. D. KAHN : I have not considered progressive waves at all.
E. N. PARKER, Enrico Fermi Institute for Nuclear Studies, University of Chicago, Chicago, Illinois: Kahn ended up by suggesting that these clouds may have lumps in them before they are ionized. Thus, you suggest that these lumps will shield the matter behind them, so that it is not ionized. If this is the case, and if one laid a ruler along the sides of the dark lanes, the rulers should all converge on the exciting star. Is that right?

F. D. KAHN : You get a shadow region beyond any lump of neutral matter, but some radiation will leak around the lump from recombinations elsewhere, and it is, therefore, very complicated really to get the exact shape.

J. A. FAY : I would add a few comments to Kahn's paper. $\mathrm{He}$ is describing what I would call an astronomical flame; that is, a wave propagation in which the energy is supplied not by chemical reaction as in ordinary flames but by absorption of radiation. In the laboratory, it is quite commonly observed that such combustion waves are unstable and have fronts which look very much like the type of fronts observed in the astronomical photographs, with prominent points directed into the reacted mixture. [Compare two photographs presented by F. N. Frenkiel at the Second Symposium (1953), Proceedings p. 160.] As I understand Kahn's instability analysis, it corresponds very closely to the instability analysis one applies to flames. One other interesting point is that we also observe fronts of this shape when such waves propagate into turbulent mixtures, in which case the scale of the disturbance is 
determined by the scale of the turbulence in the cold gas. In fact, in certain cases one can observe islands of unburned, unreacted mixture which escape through the front and remain behind. This might have some analogy to the globules.

G. C. MCVitTIE, University of Illinois, Chicago, Illinois: A plane gas cloud expands into a vacuum at three times the speed of sound. The ionized front you treat advances at the speed of sound in the ionized medium. Is it the radiation pressure that is pushing it back and slowing it up, or what is happening?

F. D. KAHN: The situation is the following. The ionization front is traveling into neutral gas, and the ionized gas is moving at the speed of sound relative to the ionization front. The back of the ionized region recedes at four times the speed of sound relative to the ionization front if the gas behaves adiabatically as though it were monatomic. Actually, in this case, it behaves rather differently, being isothermal.

H. K. SEN, GRD, AFCRC, Hanscom Field, Bedford, Massachusetts: I was interested in Goldsworthy's remark that the ionization front and the shock front stayed close together-i.e., the distance did not increase very much. This possibly indicates some coupling between the two fronts-might it not be due physically to the circumstance that some radiation was escaping out from the radiative front and was preheating the medium? When the ionization front comes nearer to the shock front, it heats the medium, and the shock ahead moves faster. Conversely, when it slows down, this effect might be less and the shock also will slow down. This might indicate a possible coupling between the two fronts.

L. BIERMANN, Max Planck Institut für Physik, Göttingen, Germany: In Pottasch's paper, we saw that the pressure in the rim, and also that in the condensation, was supposed to be very high, about $10^{-9} \mathrm{dyne} / \mathrm{cm}^{2}$. What is supposed to balance this pressure?

F. D. KAHN : There does not need to be any balance. The pressure jump at the ionization front accounts for velocity changes there. On the side near the star, the ionized gas just streams toward the star. On the remote side, a shock wave will develop in the neutral gas.

R. LANDSHOFF, Lockheed Aircraft Corporation, Sunnyvale, California: Is the radiation that we speak about here largely radiation away from the central star, or is there also some emission from the gas cloud itself?

F. D. KAHN : The assumption in all three papers is that the radiation comes entirely from the star itself. The radiation which arises in the HII region from recombinations to the ground state is neglected. There- fore, the solution given is not exactly the solution of the astronomical problem. There is a radiation field in a solid angle of about $2 \pi$ coming onto the ionization front as well, but it is rather weaker than the radiation from the star itself.

T. K. Menon, Harvard College Observatory, Cambridge, Massachusetts: Since Kahn mentioned that this treatment should be modified for the presence of small condensations, how will these considerations be modified if there is a considerable amount of dust particles in the cloud?

F. D. KAHN : Are you particularly asking about the dust or about the cool gas? One could consider a problem of this nature. A star ionizes a lumpy HI region and forms a series of bright rims. The ionization front will jump from one lump to the next as the process goes on.

T. K. MENON : On each grain, the radiation pressure would be so much more than on the neutral particles that in the initial stages probably the whole process might be controlled more by the radiation pressure than by the initial ionization process itself.

F. D. KAHN : Radiation pressure on the grains is most probably unimportant.

A. R. KANTROWITZ, Avco Research Laboratories, Everelt, Massachusetts: Looking at the photographs of the elephant trunks that were presented, one gets the impression that there is a considerable uniformity along these trunks. Now this uniformity is something that one might conceivably expect from something like the Taylor instability, where gas moves at a subsonic speed, but is it not quite difficult to understand the uniformity when one must initially suppose the existence of these globules? I would expect that one would get maybe little pieces torn off from the globules.

H. C. VAN DE HULST, Leiden Observatory, Leiden, Netherlands: I will not claim that I understand, but certainly there is a suggestion in the fact that, behind a globule with sufficient neutral gas, no ultraviolet radiation from the star can penetrate. This region in the shadow will cool by the normal cooling effects, and there will be a tendency of the surrounding gas to condense in the cool region, which then might gradually fill up.

L. SPITZER, JR., Princeton University Observatory, Princeton, New Jersey: But about one-third of the direct radiation scatters into the shadow cone because of the recombination.

H. C. VAN DE HULST : That is indeed present, but I do not see directly if this is fatal to my suggestion. 
L. SPITZER, JR. : I think it is clear that the simple analysis of the Rayleigh-Taylor instability is quite far removed from the conditions in interstellar space. On the other hand, it is not at all obvious to me that we can exclude the importance of Rayleigh-Taylor instability around an HII region. In Kahn's analysis, do I understand correctly that the condition that $J$ is constant at the inner face is tantamount to the condition that the velocity field is constant?

F. D. KAHN : If $J$ is constant, then the ionization front runs into a neutral material at a constant speed, and one gets a similarity solution.

L. SPITZER, JR.: In this case, a quasi-stationary solution exists in a frame of reference which is not accelerated, and, therefore, one would not expect any instability. So, Kahn's discussion of how $J$ usually decreases might be interpreted in the sense that deceleration instead of acceleration is to be expected around an HII region. However, if one considers not the quasi-equilibrium configuration but the actual transient solution for the outward velocity, produced when the $\mathrm{O}$ star starts shining, it is clear that there must be considerable outward acceleration of the material. I see no escape from the inference that at some stage there must be some instability, which may or may not account for what we observe.

F. D. KAHN : If the radiation keeps increasing for a short period then you get very short wavelengths for maximum instability. In fact, if you reduce the period of increase to a thousand years, this will be $10^{-5} \mathrm{pc}$. In that case, it is hardly permissible to use this analysis, because the ionization front itself will be considerably thicker than $10^{-5} \mathrm{pc}$. On the other hand, the slow increase just cannot be maintained. That is the trouble.
H. D. GREYBER, General Electric Research Laboratory, Schenectady, New York: In regard to the RayleighTaylor instability, I would remark that if there are magnetic fields of any size in the HII region, you should consider this as a plasma and get the hydromagnetic Rayleigh-Taylor instability. If this evolved roughly in the same way, the pressure of the magnetic field would, of course, act somewhat as a stabilizing influence. This should be considered when estimating rates, etc.

L. SPITZER, JR. : In that case, the appearance might be somewhat different, because two-dimensional ridges would be expected to appear rather than the usual three-dimensional cylinders. If the magnetic lines of force are parallel to the interface, the lines of force tend to move without bending.

H. D. GREYBER : True. The point came to my mind, but I did not mention it because the magnetic field might be turbulent and not remain uniform over the wavelength of a perturbation.

E. SChATZMAN : Do you have to take into account the entropy wave behind the ionization front?

F. D. KAHN : No. In considering the motion of the ionized gas, I just assumed that there was a law of the type $p \rho^{-\Gamma}=$ const and worked out the two cases where $\Gamma=5 / 3$ and $\Gamma=1$.

E. SchatZMAN : But is the constant the same everywhere? If it is not the same everywhere, you have an entropy wave.

F. D. KAHN : When the intensity of radiation reaching the ionization front remains constant, $p \rho^{-\Gamma}$ also remains constant there. 